\title{
Investigation of aquaporins and apparent diffusion coefficient from ultra-high b- values in a rat model of diabetic nephropathy
}

Yu Wang ${ }^{1}$, Heng Zhang ${ }^{1}$, Ruzhi Zhang ${ }^{1}$, Zhoushe Zhao ${ }^{2}$, Ziqian Xu ${ }^{1}$, Lei Wang ${ }^{1}$, Rongbo Liu ${ }^{1 *}$ and Fabao Gao ${ }^{1 *}$

\begin{abstract}
Background: To assess kidney damage in a rat model of type-2 diabetic nephropathy based on apparent diffusion coefficient (ADC) data obtained from ultra-high b-values and discuss its relationship to the expression of aquaporins (AQPS).

Methods: This study was approved by the institutional Animal Care and Use Committee. Thirty male SpragueDawley rats were randomised into two groups: (1) untreated controls and (2) diabetes mellitus (DM). All rats underwent diffusion-weighted imaging (DWI) with $18 \mathrm{~b}$-values $\left(0-4500 \mathrm{~s} / \mathrm{mm}^{2}\right)$. Maps of low ADC (ADC low), standard $A D C\left(A D C_{s t}\right)$ and ultra-high $A D C\left(A D C_{u h}\right)$ were calculated from low b-values $\left(0-200 \mathrm{~s} / \mathrm{mm}^{2}\right)$, standard b-values (300-1500 s/mm ${ }^{2}$ ) and ultra-high b-values $\left(1700-4500 \mathrm{~s} / \mathrm{mm}^{2}\right)$, respectively. The expression of AQPs in the kidneys was studied using immunohistochemistry. Laboratory parameters of diabetic and kidney functions, $A D C_{\text {low, }}$ $\mathrm{ADC}_{\mathrm{st}}, \mathrm{ADC}_{\mathrm{uh}}$, and the optical density (OD) of AQP expression in the two groups were compared using an independent $t$ test. Correlations between ADCs and the OD of AQP expression were evaluated by Pearson's correlation analysis.

Results: $A D C_{u h}$ were significantly higher in the cortex (CO), outer stripe of the outer medulla (OS) and inner stripe of the outer medulla (IS), and the OD values of AQ-2 were significantly higher in the OS, IS and inner medulla (IM) in DM animals compared with control animals. $A D C_{u h}$ and $O D$ values of AQP-2 expression were positively correlated in the OS, IS and IM of the kidney.
\end{abstract}

Conclusions: $A D C_{u h}$ may work as useful metrics for early detection of kidney damage in diabetic nephropathy and may be associated with AQP-2 expression.

Keywords: Diffusion-weighted imaging (DWI), Diabetic nephropathy, Aquaporins, Apparent diffusion coefficient (ADC), Ultra-high b-values

\section{Keypoints}

- Diffusion-weighted imaging (DWI) with ultra-high b-values may be valuable for the detection of diabetic nephropathy

- Apparent diffusion coefficient (ADC) calculated using ultra-high b-values is different between diabetes mellitus (DM) and control animals

\footnotetext{
* Correspondence: medicalimage@163.com; gaofabao@yahoo.com ${ }^{1}$ Department of Radiology, West China Hospital of Sichuan University, No. 37, Guoxue Lane outside the southWuhou District, Chengdu, China Full list of author information is available at the end of the article
}

- $\mathrm{ADC}_{\mathrm{uh}}$ may be associated with water transportation by aquaporins

\section{Background}

As a global health problem that affects multiple organs of the body, diabetes mellitus (DM) is known to cause proteinuria, nephrotic syndrome in the kidney, and may ultimately result in renal failure [1]. A high urinary glucose level induces elevated urinary osmotic pressure, which in turn reduces renal water reabsorption, and finally leads to polyuria [2]. Although persistent polyuria is reported to cause severe dehydration, shock from 
serious dehydration rarely develops in diabetic patients. It is, therefore, believed that the kidney has a compensating mechanism that alleviates diabetic dehydration. Water channels or aquaporins (AQPs) are membraneassociated proteins that regulate transcellular water movement across the cell membrane. So far, at least 13 mammalian AQPs (AQP 0-12) have been identified, eight of which are constitutively expressed in the kidney [3] and play a vital role in the reabsorption of water from the renal tubular fluid [4].

Several recent studies have used diffusion-weighted imaging (DWI) methods to evaluate renal function in diabetic nephropathy [5-7]. However, their values of the apparent diffusion coefficient (ADC) obtained from standard bvalues $\left(0,1000 \mathrm{~s} / \mathrm{mm}^{2}\right)$ do not differentiate early DM patients from controls in a consistent manner. Through linear regression, images taken at various b-values can be used to calculate the ADC in a particular region of interest. However, ADC estimates are susceptible to the impact of physiological changes in vivo, such as tubular flow and vascular flow, because true water diffusion contributes mainly to the signal acquired with b-values larger than approximately $200 \mathrm{~s} / \mathrm{mm}^{2}$ whereas the signal from blood perfusion with a rapid flow in tubular and vascular tubes disappears together with b-values greater than $200 \mathrm{~s} / \mathrm{mm}^{2}$ [8]. Recently, researchers who have used ultra-high b-valueDWI in some diseases suggested that ADC, when calculated using ultra-high b-values $\left(\mathrm{ADC}_{\mathrm{uh}}\right)$, may help to reveal $\mathrm{AQP}$ expression [9]. Thus, the question is: Can the slower water diffusion through the AQPs be quantified by DWI?

Therefore, this study, was aimed at assessing kidney damage in $\mathrm{DM}$ to verify the association between $\mathrm{ADC}_{\mathrm{uh}}$ and the permeability of AQPs in the cell membrane.

\section{Methods}

\section{Animals and DM induction}

All the experiments were conducted according to the guidelines of the Animal Care and Use Committee of Sichuan University, Chengdu, China and the Animal Ethics Committee Guidelines of the Animal Facility of the West China Hospital, Chengdu, China and had been given prior approval by the Experimental Animal Management Committee of Sichuan University, Chengdu, China under Contract 2016007A. Two groups of male Sprague-Dawley rats (Dashuo, Chengdu, China) were examined by magnetic resonance imaging (MRI): (1) untreated controls $(n=15)$ and (2) diabetic animals $(n=15)$. At the age of 6 to 7 weeks (weighing 180-200 g), the animals were fed a high-sucrose and high-fat diet (SCXK2014-028 Research Diet). Four weeks after the diet change, at the age of 10 to 11 weeks, DM was induced in group- 2 animals by an intraperitoneal injection of a low dose of streptozotocin (STZ) $(40 \mathrm{mg} / \mathrm{kg}$ Meilune). After the STZ treatment, 15 animals in the DM group that developed diabetes with a hyperglycemia of $>16.7 \mathrm{mmol} / \mathrm{lr}$ underwent MRI.

\section{Physiological and laboratory parameters}

General parameters, such as body weight and food and water intake, were monitored regularly. Forty days after diabetes induction, urine was collected over a period of $24 \mathrm{~h}$ and urinary albumin and creatinine concentrations were quantified. Blood was collected from the tail vein. Serum glucose, urea, albuminuria and creatinine were determined using a clinical analyser (cobas c311, Roche, Basel, Switzerland). Creatinine clearance was calculated from serum and urinary creatinine concentrations and the urine volume, which was collected over a period of $24 \mathrm{~h}$.

\section{MRI protocol}

Forty days after DM induction, all the animals underwent MRI examination using a 7.0-T small-animal scanner (Bruker Biospec 70/30, Ettlingen, Germany) and a rat heart array coil (Bruker Z114784). The animals were anaesthetised with $2 \%$ isoflurane/oxygen mixture throughout the MRI examination. The body temperature was kept constant at $37^{\circ} \mathrm{C}$ using a heating blanket monitored with a rectal temperature probe. The respiration was monitored and kept constant at between 35 to 45 breaths per min throughout the examination. Respiratory motion was reduced by imaging the animals in the supine position and with respiratory trigger. Standard T2-weighted imaging was performed in axial and oblique coronal planes (repetition time/echo time 2032/27 ms; number of excitations 4; matrix $256 \times 256$; field of view $60 \times 60 \mathrm{~mm}^{2}$; slice thickness $1.5 \mathrm{~mm}$.) The coronal plane was orientated along the long axis of the kidney. To assess the diffusion, fat-saturated echo planar diffusion-map sequences were acquired (repetition time/echo time $800 / 30 \mathrm{~ms}$; number of excitations 4; matrix $128 \times 128$; field of view $60 \times$ $60 \mathrm{~mm}^{2}$; slice thickness $1.5 \mathrm{~mm}$; three slices; b-values 0 , 50,100, 150, 200, 300, 500, 800, 1000, 1300, 1500, 1700, 2000, 2500, 3000, 3500, 4000 and $4500 \mathrm{~s} / \mathrm{mm}^{2}$ ). Scan times for the T2-weighted sequence and the diffusionmap sequence were about $4 \mathrm{~min}$ and $5 \mathrm{~min}$, respectively. The total scan time of the whole examination was approximately $16 \mathrm{~min}$.

\section{MRI data analysis}

Parameter maps of ADC were calculated using a monoexponential fit. The images were analysed by a radiologist blinded to the laboratory findings on an external workstation using Bruker software (ParaVision 5.0pl3 in/ opt/PV5.0). The renal cortex (CO), the outer stripe of the outer medulla (OS), the inner stripe of the outer medulla (IS) and the inner medulla (IM) were identified as previously described [10]. On $b=0$ images (which is essentially a T2-weighted image), regions of interest were 
placed onto the four anatomical layers of the kidney and copied to the ADC maps (Fig. 1). Maps of low ADC $\left(\mathrm{ADC}_{\text {low }}\right)$, standard $\mathrm{ADC}\left(\mathrm{ADC}_{\mathrm{st}}\right)$ and $\mathrm{ADC}_{\mathrm{uh}}$ were calculated from low b-values $\left(0-200 \mathrm{~s} / \mathrm{mm}^{2}\right)$, standard b-values $\left(300-1500 \mathrm{~s} / \mathrm{mm}^{2}\right)$ and ultra-high b-values (1700-4500 s/ $\mathrm{mm}^{2}$ ), respectively. Mean ADC values were determined respectively for each of the anatomical layers in all the animals.

\section{Renal immunohistochemistry}

Kidney tissue samples (DM $n=15$; controls $n=15$ ) from the rats were obtained immediately after MRI. The rats were euthanised and underwent fixation via abdominal aortic perfusion with $4 \%$ paraformaldehyde in $1 \%$ phosphatebuffered saline. Coronal kidney sections (from 2 to $3 \mathrm{~mm}$ thick) were fixed overnight and embedded in paraffin. The paraffin sections were cut on a sliding microtome at $4 \mu \mathrm{m}$ per section.

The sections were stained for AQP-1, AQP-2 and AQP-4 using immunohistochemical staining with antiAQP-1 antibodies (ab15080, Abcam, Shanghai, China), anti-AQP-2 antibodies (ab15081, Abcam, Shanghai, China) and anti-AQP-4 antibodies (ab9512, Abcam, Shanghai, China), respectively. After accepting the antigen retrieved by using citrate antigen retrieval solution (P0081, Beyotime, Shanghai, China), the tissue sections were treated with $3 \%$ hydrogen peroxide to inactivate endogenous peroxidase. The sections were then incubated in a 1:20 goat serum for $30 \mathrm{~min}$, rinsed, and incubated overnight at $4{ }^{\circ} \mathrm{C}$ with a 1:200 dilution of primary antibodies, including anti-AQP-1 antibodies, anti-AQP-2 antibodies and anti-AQP-4 antibodies. After three washes with phosphate-buffered saline, the sections were incubated for $90 \mathrm{~min}$ with biotinylated goat anti-rabbit immunoglobulin G antibody (SP-9001; Zhongshan Golden Bridge Biotechnology, Beijing, China). The sections underwent another three washes with phosphate-buffered saline and were then incubated with avidin-biotinylated horseradish peroxidase (SP-9001; Zhongshan Golden Bridge Biotechnology, Beijing, China) for $90 \mathrm{~min}$. The sections were rewashed three times in phosphate-buffered saline and then incubated with diaminobenzidine and hydrogen peroxide (ZLI-9018; Zhongshan Golden Bridge Biotechnology, Beijing, China). The nucleus was stained with hematoxylin. The sections were then rinsed in water for 10 min, dehydrated and covered with a coverslip for microphotography.

High-powered images ( $\times 400$ magnification) of the four anatomical layers of each kidney were taken separately (Leica ICC50 HD, Wetzlar, Germany). To analyse AQP1, AQP-2 and AQP-4 staining, the regions covered by AQP-1, AQP-2 and AQP-4 staining were calculated by optical density (OD) [11]. We measured the OD values three times and averaged the results.
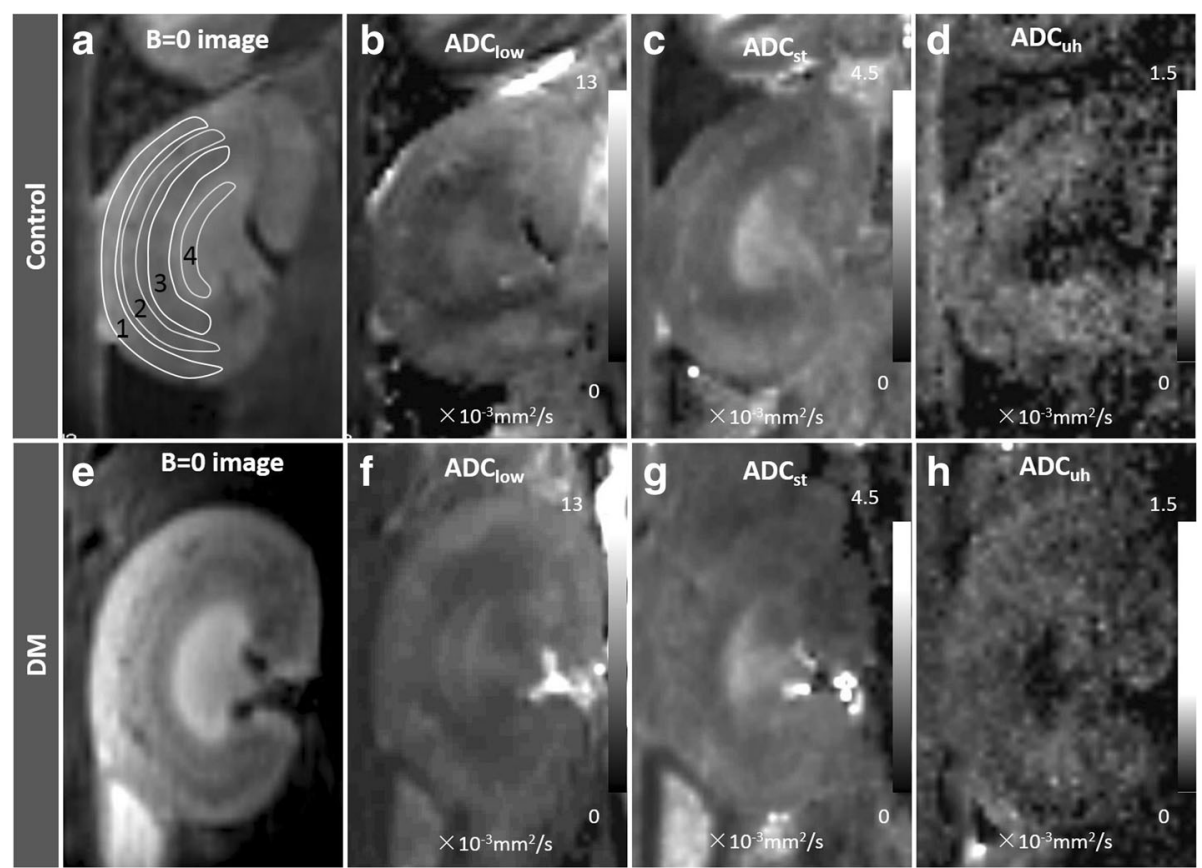

Fig. 1 Example of diffusion maps in an animal with streptozotocin-induced diabetes mellitus (DM) and a control animal. $\mathbf{a}-\mathbf{d}, b=0$ image, $A D C_{\text {low }}$ $A D C_{\text {st }}$ and $A D C_{\text {uh }}$ maps in a $D M$ animal. e-h, $b=0$ image, $A D C_{\text {low, }} A D C_{\text {st }}$ and $A D C_{\text {uh }}$ maps in a control animal. On $b=0$ images, the four anatomical layers of the kidney can be distinctively observed. In these diffusion maps, regions of interest were placed onto the cortex (1), the outer stripe of the outer medulla (2), the inner stripe of the outer medulla (3) and the inner medulla (4) as exemplarily demonstrated in $\mathbf{a}$. In DM animals, ADCun increased apparently in CO, OS and IS compared with controls (panels $\mathbf{d}$ and $\mathbf{h}$ ) 


\section{Statistical analysis}

Statistical analysis was performed with SPSS 19.0 (SPSS Inc., Chicago, IL, USA). Quantitative data were tested for normal distribution by using the Kolmogorov-Smirnov test. Normally distributed data were expressed as mean \pm standard deviation. Laboratory parameters of diabetic and kidney functions, $\mathrm{ADC}_{\mathrm{low}}, \mathrm{ADC}_{\mathrm{st}}, \mathrm{ADC}_{\mathrm{uh}}$, and the $\mathrm{OD}$ of AQPs expression in the two groups were evaluated with an independent $t$ test with equal variance.. Correlation analysis between ADCs and OD values of AQPs of the anatomical layers was performed by Pearson's correlation analysis. To avoid the introduction of type-I errors, Bonferroni's correction was applied to the preset level of significance $(p<0.050)$. The significance levels were, therefore, as follows: $p<0.0083(0.05 / 6)$ in Table $1 ; p<$ $0.017(0.05 / 3)$ in Tables 2,3 and 4.

\section{Results}

\section{Physiological and laboratory parameters}

Streptozotocin-induced DM reduced bodyweight gain but increased serum glucose in the DM animals compared with controls $(p<0.001$; Table 1$)$. In DM animals, creatinine clearance was no difference compared with controls $(p=0.048)$ but the urinary output of the diabetic group was higher than that of controls by 14fold. In addition, serum urea elevated significantly in the DM group compared with controls $(p=0.008)$. Urine albumin levels of the DM group, however, were reduced in comparison with that of controls $(p=$ 0.007; Table 1).

\section{DWI}

All the MRI studies $(n=30)$ were included in the analysis. MRI could identify the four anatomical layers of the kidney (CO, OS, IS and IM) in all the animals (Fig. 1). An example of $\mathrm{ADC}_{\text {low }}, \mathrm{ADC}_{\mathrm{st}}$ and $\mathrm{ADC}_{\mathrm{uh}}$ maps in both a DM animal and a control animal is given in Fig. 1. $\mathrm{ADC}_{\mathrm{uh}}$ of the CO, OS and IS in the DM animals was significantly higher than that in controls (Table 2).

Table 1 Characteristics of the two groups

\begin{tabular}{llll}
\hline & Controls & DM & $p$ value \\
\hline Body weight $(\mathrm{g})$ & $409 \pm 23$ & $286 \pm 41$ & $<0.001$ \\
Blood glucose $(\mathrm{mmol} / \mathrm{l})$ & $5.8 \pm 0.7$ & $28 \pm 4.1$ & $<0.001$ \\
Creatinine clearance $(\mathrm{ml} / \mathrm{min})$ & $1.5 \pm 0.7$ & $2.2 \pm 1.1$ & 0.048 \\
Serum urea $(\mathrm{mmol} / \mathrm{l})$ & $6.7 \pm 0.6$ & $9.7 \pm 4.0$ & 0.008 \\
Urine output $\mathrm{ml} / 24 \mathrm{~h}$ & $9.5 \pm 2.2$ & $134 \pm 21$ & $<0.001$ \\
Urine albumin $\mathrm{mg} / \mathrm{l}$ & $2.5 \pm 1.0$ & $1.5 \pm 0.7$ & 0.007 \\
\hline
\end{tabular}

Data are presented as mean \pm standard deviation. Body weight and laboratory parameters were obtained after the day of the magnetic resonance imaging (MRI) scan. Comparisons between the diabetic group (DM) and controls were made by using an independent $t$ test. Based on Bonferroni's correction, $p<0.0083(0.05 / 6)$ was considered to indicate a statistically significant difference
Table 2 Apparent diffusion coefficients (ADCs) in diabetes mellitus (DM) animals and controls

\begin{tabular}{|c|c|c|c|c|}
\hline Position & $\begin{array}{l}\text { Parameter } \\
\left(\times 10^{-3} \mathrm{~s} / \mathrm{mm}^{2}\right)\end{array}$ & Controls & $D M$ & $p$ value \\
\hline \multirow[t]{3}{*}{$\mathrm{CO}$} & $A D C_{\text {low }}$ & $2.64 \pm 0.525$ & $2.52 \pm 0.545$ & ns, 0.392 \\
\hline & $A D C_{s t}$ & $1.22 \pm 0.230$ & $1.30 \pm 0.228$ & $n s, 0.220$ \\
\hline & $A D C_{u h}$ & $0.196 \pm 0.078$ & $0.262 \pm 0.050$ & $<0.001$ \\
\hline \multirow[t]{3}{*}{ OS } & $A D C_{\text {low }}$ & $2.56 \pm 0.543$ & $2.36 \pm 0.519$ & ns, 0.146 \\
\hline & $A D C_{s t}$ & $1.27 \pm 0.226$ & $1.31 \pm 0.243$ & $n s, 0.501$ \\
\hline & $A D C_{u h}$ & $0.190 \pm 0.088$ & $0.240 \pm 0.067$ & 0.016 \\
\hline \multirow[t]{3}{*}{ IS } & $A D C_{\text {low }}$ & $2.33 \pm 0.636$ & $2.15 \pm 0.536$ & $n s, 0.250$ \\
\hline & $A D C_{s t}$ & $1.29 \pm 0.275$ & $1.22 \pm 0.218$ & ns, 0.316 \\
\hline & $A D C_{u h}$ & $0.203 \pm 0.081$ & $0.268 \pm 0.096$ & 0.006 \\
\hline \multirow[t]{3}{*}{ IM } & $A D C_{\text {low }}$ & $2.77 \pm 0.561$ & $2.66 \pm 0.550$ & ns, 0.447 \\
\hline & $A D C_{s t}$ & $1.67 \pm 0.305$ & $1.60 \pm 0.317$ & ns, 0.405 \\
\hline & $A D C_{u h}$ & $0.140 \pm 0.066$ & $0.190 \pm 0.101$ & ns, 0.027 \\
\hline
\end{tabular}

Data are presented as mean \pm standard deviation. Statistical analysis was performed using an independent $t$ test between the two groups. DM diabetes mellitus, CO cortex, OS outer stripe of the outer medulla, IS inner stripe of the outer medulla, $I M$ inner medulla, $A D C_{\text {low }} A D C$ calculated using the low b-values, $A D C_{s t} A D C$ calculated using the standard $\mathrm{b}$-values, $A D C_{u h} A D C$ calculated using the ultra-high b-values, ns not significant. Based on Bonferroni's correction, $p<$ $0.017(0.05 / 3)$ was considered to indicate a statistically significant difference

Although $\mathrm{ADC}_{\mathrm{uh}}$ of the IM in the $\mathrm{DM}$ animals was significantly higher than that in controls at $p<0.050$ level, there was no significant difference after Bonferroni's correction. However, no significant difference was found in $\mathrm{ADC}_{\text {low }}$ and $\mathrm{ADC}_{\mathrm{st}}$ in each region of interest between the two groups (Table 2).

Table 3 Optical density of aquaporins (APQs) positively stained on different anatomical layers of the kidney in diabetes mellitus (DM) animals and controls

\begin{tabular}{|c|c|c|c|c|}
\hline Position & $\mathrm{AQP}$ & Controls & DM & $p$ value \\
\hline \multirow[t]{3}{*}{$\mathrm{CO}$} & AQP-1 & $0.050 \pm 0.013$ & $0.049 \pm 0.017$ & $n s, 0.818$ \\
\hline & AQP-2 & $0.026 \pm 0.011$ & $0.035 \pm 0.010$ & ns, 0.025 \\
\hline & AQP-4 & $0.014 \pm 0.006$ & $0.012 \pm 0.006$ & $n s, 0.504$ \\
\hline \multirow[t]{3}{*}{ OS } & AQP-1 & $0.056 \pm 0.011$ & $0.048 \pm 0.014$ & ns, 0.095 \\
\hline & AQP-2 & $0.045 \pm 0.011$ & $0.060 \pm 0.019$ & 0.009 \\
\hline & AQP-4 & $0.011 \pm 0.003$ & $0.009 \pm 0.004$ & $n s, 0.234$ \\
\hline \multirow[t]{3}{*}{ IS } & AQP-1 & $0.042 \pm 0.010$ & $0.039 \pm 0.009$ & ns, 0.341 \\
\hline & AQP-2 & $0.036 \pm 0.009$ & $0.058 \pm 0.016$ & 0.000 \\
\hline & AQP-4 & $0.020 \pm 0.005$ & $0.019 \pm 0.008$ & ns, 0.644 \\
\hline \multirow[t]{3}{*}{ IM } & AQP-1 & $0.036 \pm 0.010$ & $0.031 \pm 0.009$ & ns, 0.140 \\
\hline & AQP-2 & $0.027 \pm 0.010$ & $0.040 \pm 0.011$ & 0.002 \\
\hline & AQP-4 & $0.026 \pm 0.008$ & $0.025 \pm 0.009$ & ns, 0.694 \\
\hline
\end{tabular}

Data are presented as mean \pm standard deviation. Statistical analysis was performed using an independent $t$ test between the two groups. DM diabetes mellitus, DM diabetes mellitus, CO cortex, OS outer stripe of the outer medulla, IS inner stripe of the outer medulla, IM inner medulla $n s$, not significant. Based on Bonferroni's correction, $p<0.017(0.05 / 3)$ was considered to indicate a statistically significant difference 
Table 4 Correlation between apparent diffusion coefficients (ADCs) and aquaporin-2 (AQP-2) in diabetes mellitus (DM) animals at four anatomical layers of the kidney

\begin{tabular}{|c|c|c|c|c|c|c|c|}
\hline \multirow[t]{2}{*}{ Position } & \multirow[t]{2}{*}{$\mathrm{AQP}$} & \multicolumn{2}{|l|}{$A D C_{\text {low }}$} & \multicolumn{2}{|l|}{$\mathrm{ADC}_{\mathrm{st}}$} & \multicolumn{2}{|l|}{$A D C_{u h}$} \\
\hline & & $r$ & $p$ & $r$ & $p$ & $r$ & $p$ \\
\hline \multirow[t]{3}{*}{$\mathrm{CO}$} & AQP-1 & -0.123 & ns 0.509 & 0.026 & ns 0.890 & -0.023 & ns, 0.903 \\
\hline & AQP-2 & -0.178 & ns 0.346 & -0.044 & ns 0.871 & 0.427 & $n s, 0.018$ \\
\hline & AQP-4 & -0.109 & ns 0.565 & -0.098 & ns 0.607 & -0.174 & $n s, 0.358$ \\
\hline \multirow[t]{3}{*}{ OS } & AQP-1 & 0.123 & ns 0.516 & 0.141 & ns 0.458 & -0.157 & ns, 0.407 \\
\hline & $A Q P-2$ & -0.280 & ns 0.134 & -0.047 & ns 0.806 & 0.598 & 0.000 \\
\hline & $A Q P-4$ & -0.193 & ns 0.306 & -0.139 & ns 0.464 & -0.194 & $n s, 0.304$ \\
\hline \multirow[t]{3}{*}{ IS } & $A Q P-1$ & -0.266 & ns 0.155 & -0.106 & ns 0.576 & -0.026 & $n s, 0.893$ \\
\hline & AQP-2 & -0.277 & ns 0.138 & -0.139 & ns 0.464 & 0.490 & 0.006 \\
\hline & $A Q P-4$ & -0.073 & ns 0.700 & -0.036 & ns 0.850 & 0.140 & $n s, 0.460$ \\
\hline \multirow[t]{3}{*}{ IM } & AQP-1 & 0.175 & ns 0.355 & 0.293 & ns 0.115 & -0.039 & ns, 0.838 \\
\hline & $A Q P-2$ & -0.109 & ns 0.567 & 0.003 & ns 0.986 & 0.451 & 0.012 \\
\hline & $A Q P-4$ & 0.141 & ns 0.459 & 0.173 & ns 0.362 & -0.227 & $n s, 0.227$ \\
\hline
\end{tabular}

The $r$ and $p$ values were obtained using Pearson's correlation analysis. DM diabetes mellitus, DM diabetes mellitus, CO cortex, OS outer stripe of the outer medulla, IS inner stripe of the outer medulla, IM inner medulla $n s$, not significant. Based on Bonferroni's correction, $p<0.017$ ( $0.05 / 3$ tests) was considered to indicate a statistically significant difference

\section{Immunohistochemistry}

In both $\mathrm{DM}$ and control kidney sections were found AQP-1, AQP-2 and AQP-4 immunoreactive cells (Fig. 2). AQP-1 staining and AQP-2 staining were prominent and widespread in different anatomical layers of the kidney in the diabetic rats. The OD value of AQP-2 expression in the OS, IS and IM in diabetic animals was significantly higher than that in controls (Table 3). Although the OD value of AQP-2 expression in $\mathrm{CO}$ in the DM animals was significantly higher than that in controls at $p<0.050$ level, there was no significant difference after Bonferroni's correction. However, there was no significant difference between the two groups in their OD values of AQP-1 and AQP-4 expression (Table 3).

\section{Correlation between ADC values and AQP-2}

$\mathrm{ADC}_{\mathrm{uh}}$ values were positively correlated with $\mathrm{OD}$ values of AQP-2 expression in the OS, IS and IM of the kidney (Table 4, Fig. 3). Although there were positive correlations between $\mathrm{ADC}_{\mathrm{uh}}$ and $\mathrm{OD}$ values of AQP-2 expression in $\mathrm{CO}$ at the $p<0.050$ level, there was no significant difference after Bonferroni's correction. The $\mathrm{ADC}_{\mathrm{low}}$ and $\mathrm{ADC}_{\mathrm{st}}$ values on the four anatomical layers of the kidney were not correlated with the OD values of AQP-2 expression. In addition, there were no correlations between ADCs and OD values of AQP-1 and AQP-4 expression (Table 4).

\section{Discussion}

We used an STZ-induced model of diabetes which used a high-fat diet combined with a low STZ dose. The animals developed features of type- 1 and type- 2 DM with reduced insulin production and symptoms of a metabolic syndrome as previously described [12]. All the high-fat-diet and STZtreated rats developed typical hyperglycaemia, increased urine output, and loss of body weight as typical symptoms of diabetes. In our diabetic model of animals, serum urea increased significantly, which is consistent with previous studies [7].

During the progression of diabetic nephropathy, the primary pathological changes involved fibrosis, impaired renal function, as well as chronic kidney diseases, all of which are known to be associated with a decrease in ADC both in human and in animal studies [13-15]. However, in our study, $\mathrm{ADC}_{\text {low }}$ and $\mathrm{ADC}_{\text {st }}$ of $\mathrm{DM}$ animals and control animals did not show any significant change. These results are in accordance with those of Cakmak et al. [5], who reported no significant difference in $\mathrm{ADC}$ values between the control group and earlier diabetic stages (stage 1 and stage 2). In an experimental rat diabetic nephropathy study by Ries et al. [6], no statistically significant difference was found between CO, IS and IM except for a subgroup of diabetic animals with oedematous cellular damage. In a study by Hueper et al. [7], the ADC values of the DM animals and controls were not different in their medulla and cortex. Interestingly, they found that medullary and cortical ADC was even elevated in diabetic animals with unilateral nephrectomy, which they explained by using low b-values of 0 and $300 \mathrm{~s} / \mathrm{mm}^{2}$. However, if $\mathrm{ADC}_{\text {low }}$ values were sensitive enough to the perfusion change within the kidney, higher $\mathrm{ADC}_{\text {low }}$ values (due to hyperfiltration in the diabetic kidneys) should have been found in our study. The increased ADC in DM with unilateral nephrectomy in the study by Hueper et al. [7] was caused not only by 


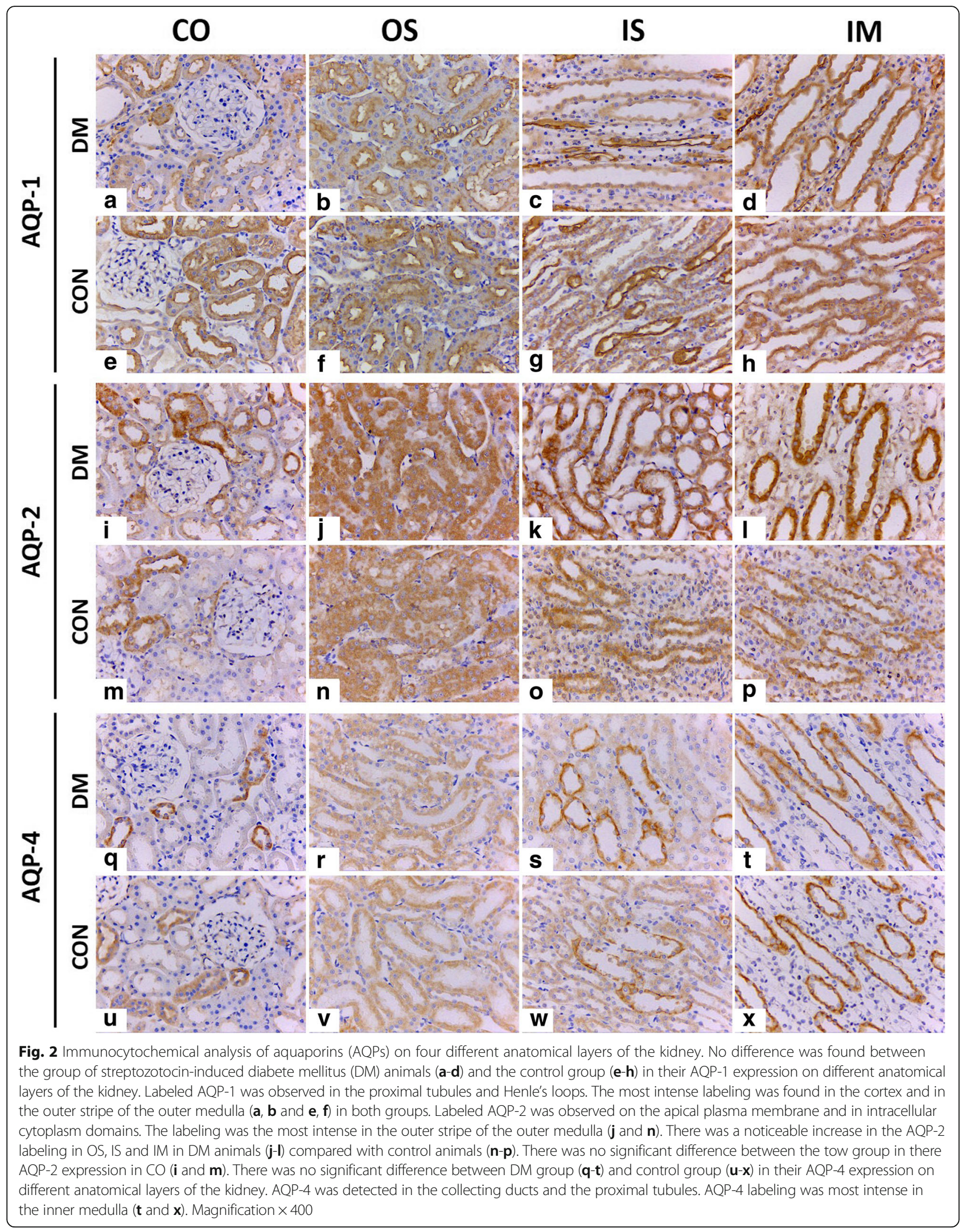



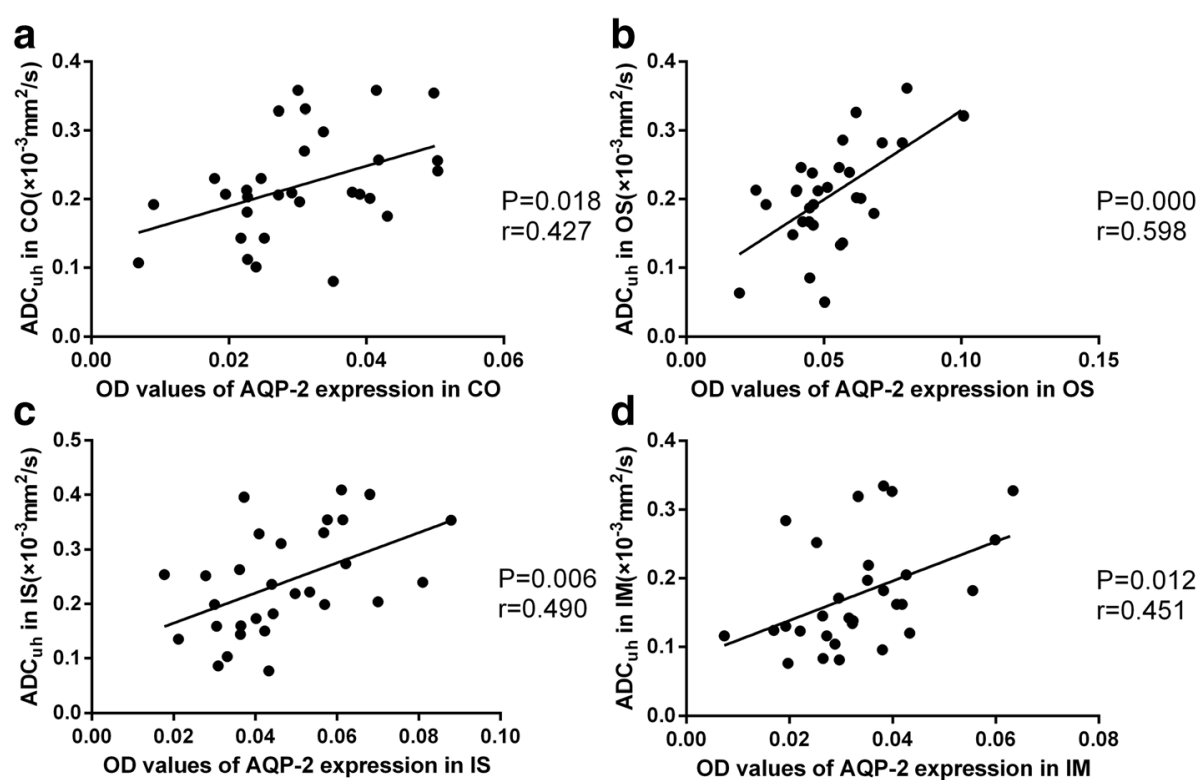

Fig. 3 Correlation between renal optical density (OD) values of aquaporin-2 (AQP-2) expression and values of apparent diffusion coefficient obtained

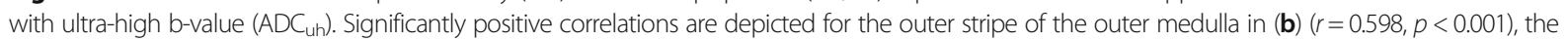
inner stripe of the outer medulla in (c) $(r=0.490, p=0.006)$ and the inner medulla in (d) $(r=0.451, p=0.012)$. Although there was a positive correlation between ADCuh and OD values of AQP-2 expression in cortex in (a) $(r=0.427, p=0.018)$, there was no significant difference after Bonferroni's correction

hyperfiltration but also by a compensatively increased renal blood flow due to unilateral nephrectomy. Therefore, our results indicated that $\mathrm{ADC}_{\mathrm{low}}$ and $\mathrm{ADC}_{\mathrm{st}}$ measurements seem to reflect histopathological changes within the tissue not sensitive to the energy metabolism or the tubular flow or reabsorption.

We further suggest that $\mathrm{ADC}_{\mathrm{uh}}$ may serve as a promising biometric for the early detection of diabetic nephropathy. Moreover, $\mathrm{ADC}_{\mathrm{uh}}$ was lower than the $\mathrm{ADC}_{\mathrm{st}}$ and $\mathrm{ADC}_{\text {low }}$ values, which corresponds with the finding that the higher $\mathrm{b}$-value in the scan, the lower the estimated ADC values in the kidney [16]. ADC contained information not only about diffusion but also about perfusion and flow effects. Some studies [17] suggested that diffusion signal intensity with low b-values is mainly contributed by a fast diffusion component, as $\mathrm{ADC}_{\text {low }}$ is mainly illustrated by the fast blood perfusion in the kidney. Diffusion signal intensity with high b-values is dominated primarily by a slow diffusion component, as $\mathrm{ADC}_{\mathrm{st}}$ is related to the slow water diffusion. Thus, according to the relationship between the b-value and diffusion signal intensity, the $\mathrm{ADC}_{\mathrm{uh}}$ measured at ultrahigh b-value might have something to do with a slower diffusion component because we observed a weak $\mathrm{ADC}_{\mathrm{uh}}$ in the present study. Moreover, our findings indicate that $\mathrm{ADC}_{\mathrm{uh}}$ measurement is more sensitive than $\mathrm{ADC}_{\text {low }}$ and $\mathrm{ADC}_{\mathrm{st}}$ measurement to the energy metabolism or the tubular flow or reabsorption.

The changes we found in $\mathrm{ADC}_{\mathrm{uh}}$ may correlate with the changes in AQP expression. In this research we used immunohistochemistry to measure the expression of AQP-1, AQP-2 and AQP-4 and we found that OD values of AQP-2 expression increased in the OS, IS and IM whereas expression of AQP-1and AQP-4 in different anatomical layers of the kidney in the diabetic animals did not change compared with controls. Aquaporins play an important role in the reabsorption of water from renal tubular fluid. In one study, polyuria and the inability to concentrate their urine has been reported in AQP-1deficient mice [3]. In long-term type-1 diabetic rats, polyuria probably results from a reduced AQP-1 expression in renal tissues [18]. Earlier studies using STZ-induced animal models of DM also reported an increased AQP-2 expression in the kidneys [19-23]. Our finding agrees with the abovementioned results and supports the proposition that an increased AQP-2 expression might work as a compensatory mechanism that alleviates dehydration.

In recent studies, attention has been paid to the relationship between the ADC and AQP expression in the membranes. A clinical study using ultra-high b-valueDWI in Parkinson's disease suggests that $\mathrm{ADC}_{\mathrm{uh}}$ may help to illustrate the function of the AQP [9]. In addition, the speed of water transportation by AQPs in the membrane is about $0.45 \times 10^{-3} \mathrm{~mm} / \mathrm{s}$ slower than the water diffusion speed between cell gaps [24]. Moreover, our study showed a positive correlation between $\mathrm{ADC}_{\mathrm{uh}}$ and AQP-2 expression, even though only a limited correlation. Thus, based upon these findings, we propose that DWI-MRI with ultra-high b-values might be able to assess water transportation by AQPs. 
There are several limitations in the present study. First, we only measured at one time point during the progression of the disease. Second, the investigated animal population was relatively small. A large number of subjects and multiple time point measurements during the progression of the disease can help to confirm our findings. Third, the higher b-values we used may lead to a lower signal-to-noise ratio. However, we used a 7.0-T small-animal scanner to compensate the drawback, which may help us obtain a more powerful signal-tonoise ratio. Another limitation is that the regions of interest were drawn manually. Finally, as optical OD values of $\mathrm{AQP}$ expression were semiquantitative they may not be highly accurate because OD values are susceptible to the condition of staining and exposure duration when the images are taken. However, this drawback may be compensated by the immunohistochemistry that we used to achieve OD values, which were stained under the same condition and in the same batch. Images were taken at the same exposure duration to make sure that our data are true and comparable.

In conclusion, we found significantly higher $\mathrm{ADC}_{\mathrm{uh}}$ values in $\mathrm{CO}$, OS and IS and AQP-2 expression in the OS, IS and IM in DM animals compared with controls. Thus, we suggest that $\mathrm{AQP}-2$ plays an important role in alleviating dehydration caused by poorly uncontrolled diabetes with polyuria and that DWI with ultra-high b-values may be valuable for noninvasive early detection and discriminative diagnosis of diabetic nephropathy. Lastly, the change in $\mathrm{ADC}_{\mathrm{uh}}$ may reflect function of the $\mathrm{AQP}$.

\section{Abbreviations \\ ADC: Apparent diffusion coefficient; $A D C_{\text {low: }}$ ADC calculated using low b-values; $A D C_{\text {st: }}$ ADC calculated using standard b-values; $A D C_{u n}$ : ADC calculated using ultra- high b-values; AQP: Aquaporin; CO: Cortex; DM: Diabetes mellitus; DWl: Diffusion- weighted imaging; IM: Inner medulla; IS: Inner stripe of the outer medulla; OD: Optical density; OS: Outer stripe of the outer medulla; STZ: Streptozotocin}

\section{Acknowledgements}

This trial was technically supported by GE Healthcare, China. This study was partly supported by National Key Research and Development Program of China (No. 2016YFA0201400); the International Cooperation and Exchange Projects of the National Natural Science Foundation of China (No. 81520108014).

\section{Authors' contribution}

YW, HZ carried out the Imaging studies, participated in the sequence alignment and drafted the manuscript. YW, ZX and RZ carried out the immunoassays. LW participated in the sequence alignment. YW, ZS participated in the design of the study and performed the statistical analysis. FG and RL conceived of the study and participated in its design and coordination. All authors read and approved the final manuscript.

\section{Competing interests}

The authors declare that they have no competing interests.

\section{Publisher's Note}

Springer Nature remains neutral with regard to jurisdictional claims in published maps and institutional affiliations.

\section{Author details}

'Department of Radiology, West China Hospital of Sichuan University, No. 37 , Guoxue Lane outside the southWuhou District, Chengdu, China. ${ }^{2}$ General Electronic Company Healthcare (China), Beijing, China.

Received: 9 March 2017 Accepted: 12 July 2017

Published online: 10 October 2017

\section{References}

1. Lane PH, Steffes MW, Fioretto P, Mauer SM (1993) Renal interstitial expansion in insulin-dependent diabetes mellitus. Kidney Int 43:661-667

2. Spira A, Gowrishankar M, Halperin ML (1997) Factors contributing to the degree of polyuria in a patient with poorly controlled diabetes mellitus. Am J Kidney Dis 30:829-835

3. Verkman AS (2000) Physiological importance of aquaporins: lessons from knockout mice. Curr Opin Nephrol Hypertens 9:517-522

4. Kortenoeven ML, Fenton RA (2014) Renal aquaporins and water balance disorders. Biochim Biophys Acta 1840:1533-1549

5. Cakmak P, Yagci AB, Dursun B et al (2014) Renal diffusion-weighted imaging in diabetic nephropathy: correlation with clinical stages of disease. Diagn Interv Radiol 20:374-378

6. Ries M, Basseau F, Tyndal B et al (2003) Renal diffusion and BOLD MRI in experimental diabetic nephropathy. Blood oxygen level-dependent. J Magn Reson Imaging 17:104-113

7. Hueper K, Hartung D, Gutberlet M et al (2012) Magnetic resonance diffusion tensor imaging for evaluation of histopathological changes in a rat model of diabetic nephropathy. Invest Radiol 47:430-437

8. Sigmund EE, Vivier PH, Sui D et al (2012) Intravoxel incoherent motion and diffusion-tensor imaging in renal tissue under hydration and furosemide flow challenges. Radiology 263:758-769

9. Xueying L, Zhongping Z, Zhoushe Z et al (2015) Investigation of apparent diffusion coefficient from ultra-high b-values in Parkinson's disease. Eur Radiol 25:2593-2600

10. Thomas SR (2009) Kidney modeling and systems physiology. Wiley Interdiscip Rev Syst Biol Med 1:172-190

11. Abdel-Hamid GA (2013) Effect of vitamin E and selenium against aluminuminduced nephrotoxicity in pregnant rats. Folia Histochem Cytobiol 51:312-319

12. Srinivasan K, Viswanad B, Asrat L et al (2005) Combination of high-fat dietfed and low-dose streptozotocin-treated rat: a model for type 2 diabetes and pharmacological screening. Pharmacol Res 52:313-320

13. Lu L, Sedor JR, Gulani $V$ et al (2011) Use of diffusion tensor MRI to identify early changes in diabetic nephropathy. Am J Nephrol 34:476-482

14. Chen X, Xiao W, Li X et al (2014) In vivo evaluation of renal function using diffusion weighted imaging and diffusion tensor imaging in type 2 diabetics with normoalbuminuria versus microalbuminuria. Front Med 8:471-476

15. Togao O, Doi S, Kuro-o M et al (2010) Assessment of renal fibrosis with diffusionweighted MR imaging: study with murine model of unilateral ureteral obstruction. Radiology 255:772-780

16. Zhang $\mathrm{J}$, Sigmund $\mathrm{EE}$, Chandarana $\mathrm{H}$ et al (2010) Variability of renal apparent diffusion coefficients: limitations of the monoexponential model for diffusion quantification. Radiology 254:783-792

17. Notohamiprodjo M, Chandarana H, Mikheev A et al (2015) Combined intravoxel incoherent motion and diffusion tensor imaging of renal diffusion and flow anisotropy. Magn Reson Med 73:1526-1532

18. Vidotti DB, Arnoni CP, Maquigussa E, Boim MA (2008) Effect of long-term type 1 diabetes on renal sodium and water transporters in rats. Am J Nephrol 28:107-114

19. Bardoux P, Ahloulay M, Le Maout S et al (2001) Aquaporin-2 and urea transporterA1 are up-regulated in rats with type I diabetes mellitus. Diabetologia 44:637-645

20. Song J, Knepper MA, Verbalis JG, Ecelbarger CA (2003) Increased renal ENaC subunit and sodium transporter abundances in streptozotocin-induced type 1 diabetes. Am J Physiol Renal Physiol 285:F1125-F1137

21. Mozaffari MS, Abdelsayed R, Liu JY et al (2012) Renal distal tubule proliferation and increased aquaporin 2 level but decreased urine osmolality in $\mathrm{db} / \mathrm{db}$ mouse: treatment with chromium picolinate. Exp Mol Pathol 92:54-58

22. Satake M, Ikarashi $N$, Ichikawa $Y$ et al (2010) The role of renal aquaporin 2 in the alleviation of dehydration associated with diabetic polyuria in KKAy mice. Life Sci 87:475-480

23. Li C, Wang W, Summer SN et al (2006) Hyperosmolality in vivo upregulates aquaporin 2 water channel and $\mathrm{Na}-\mathrm{K}-2 \mathrm{Cl}$ co-transporter in Brattleboro rats. J Am Soc Nephrol 17:1657-1664

24. Zhu F, Tajkhorshid E, Schulten K (2004) Theory and simulation of water permeation in aquaporin-1. Biophys J 86:50-57 